\title{
Cochlear Implantation in Neurobrucellosis
}

\author{
Münir Demir Bajin ${ }^{1}$, Özden Savaş$^{2}$, Filiz Aslan $^{3}$, Levent Sennaroğlu $^{1}$ \\ ${ }^{1}$ Department of Otolaryngology, Hacettepe University Hospital, Ankara, Turkey \\ ${ }^{2}$ Department of Otolaryngology, Hacettepe University Faculty of Medicine, Ankara, Turkey \\ ${ }^{3}$ Audiology Unit, Department of Otolaryngology, Hacettepe University Hospital, Ankara, Turkey
}

Background: Neurobrucellosis is a disease consisting of a wide spectrum of complications such as peripheral neuropathy, cranial nerve involvement, ataxia, meningeal irritation, paraplegia, seizures, coma, and even death. The vestibulocochlear nerve seems to be the most commonly affected cranial nerve (10\%). We present a patient with neurobrucellosis whose auditory perception and speech intelligibility skill performances improved after cochlear implantation.

Case Report: A 35 year-old woman was admitted to another hospital 2 years ago with the symptoms of headache, nausea, and altered consciousness, who was finally diagnosed with neurobrucellosis. She developed bilateral profound sensorineural hearing loss during the following 6 months. There was no benefit of using hearing aids. After successful treatment of her illness, she was found to be suitable for cochlear implantation. After the operation, her auditory perception skills improved significantly with a Categories of Auditory Performance (CAP) score of 5. According to clinical observations and her family members' statements, her Speech Intelligibility Rating (SIR) score was 3. Her speech intelligibility skills are still improving.

Conclusion: Our case report represents the second case of hearing rehabilitation with cochlear implantation after neurobrucellosis. Cochlear implantation is a cost-effective and time-proven successful intervention in post-lingual adult patients with sensorineural hearing loss. Early timing of the surgery after appropriate treatment of meningitis helps the patient to achieve better postoperative results. Keywords: Cochlear implantation, neurobrucellosis, sensorineural hearing loss
Brucellosis is one of the most commonly seen zoonotic infections around the world. The major subtypes are B. suis, B. melitensis and B. abortus, with B. melitensis being the most common. Raw meat and unpasteurized dairy products are the major transmission routes of the disease. Thanks to current standards of care and antibiotherapy, once diagnosed, the disease itself is quite manageable and complete response is expected with low mortality. However, brucellosis is a systemic illness of diverse manifestations, putting the patients at risk of some serious major complications. Central nervous system (CNS) involvement is reported in around 5\% of patients. A recent review analyzing 35 previous reports of patients diagnosed and treated in a major endemic region of the disease revealed that neurobrucellosis consisted of a wide spectrum of complications such as peripheral neuropathy, cranial nerve involvement, ataxia, meningeal irritation, paraplegia, seizures, coma, and even death. The vestibulocochlear nerve seems to be the most commonly affected cranial nerve (10\%) (1). In this article, we present a patient with neurobrucellosis and her auditory perception and speech intelligibility skills performances after 5 months of cochlear implant use. Both her speech intelligibility and auditory perception skills improved immediately after implantation. She uses her implant regularly, every day.

\section{CASE PRESENTATION}

Our patient was a 35 year-old woman who was admitted to another hospital 2 years ago with the symptoms of headache, nausea, and altered consciousness. There was no history of 
seizure or any other neurological illness. Laboratory tests revealed elevated protein $(238 \mathrm{mg} / \mathrm{dL})$, lowered glucose levels $(18 \mathrm{mg} / \mathrm{dL})$ and a positive agglutination test for Brucellosis. She had developed bilateral profound sensorineural hearing loss during the following 6 months. There was no benefit of using hearing aids. Again, in March 2013, she had another episode of headache and stuporous state. With the confirmed diagnosis of Brucella meningitis and hearing loss, she was referred to our institution with daily maintenance therapy of rifampicin $600 \mathrm{mg}$ and 2x100 mg of doxycycline. Her lumbar

TABLE 1. Cerebrospinal fluid biochemistry

\begin{tabular}{lc}
\hline Blood $(\mathrm{mg} / \mathrm{dL})$ & CSF $(\mathrm{mg} / \mathrm{dL})$ \\
\hline Sodium: $138.4(135-145)$ & Glucose: $36(40-80)$ \\
Chloride: $105.6(95-110)$ & Chloride: $122.2(110-128)$ \\
Protein: $6.70(6.4-8.3)$ & Protein: $36(40-80)$ \\
Albumin: $4.16(3.4-4.8)$ & \\
Globulin: 2.54 & \\
\hline
\end{tabular}

CSF: cerebrospinal fluid; mg: milligram; dL: deciliter

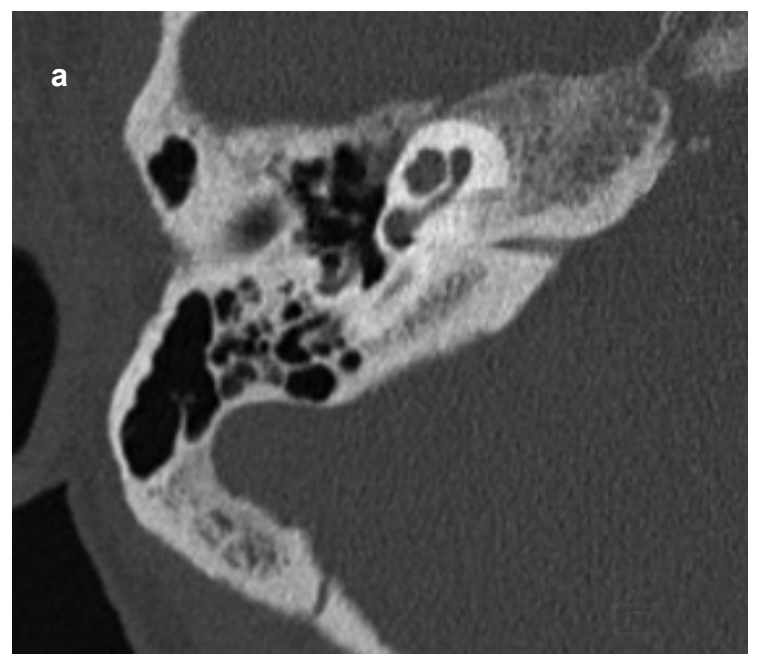

puncture showed up elevated cerebrospinal fluid (CSF) protein and decreased glucose levels (Table 1).

Her CSF Brucella agglutination titer was 1/160 (+). She was referred to the Department of Infectious Diseases and recommended 2x2 g ceftriaxone, 2x100 mg doxycycline and 1x900 $\mathrm{mg}$ rifampicin. Meanwhile, her audiological tests were repeated and confirmed. On physical examination, the external ear canal and tympanic membrane were normal in appearance for both ears and the tympanometric tests were normal. Her hearing level thresholds were higher than $110 \mathrm{~dB}$-SPL for the entire frequency spectrum, consistent with profound sensorineural hearing loss (SNHL). Her computed tomography and magnetic resonance imaging (MRI) scans for both ears were normal without any sign of labyrinthitis ossificans (Figure 1,2). Her cranial MRI revealed diffuse dural thickening and minimal diffuse pial contrast enhancement (Figure 3). Due to the active disease process, despite being a good candidate for cochlear implantation, her operation had to be postponed. In April 2013, she was discharged from the hospital with the same oral drug regimen as a maintenance therapy.

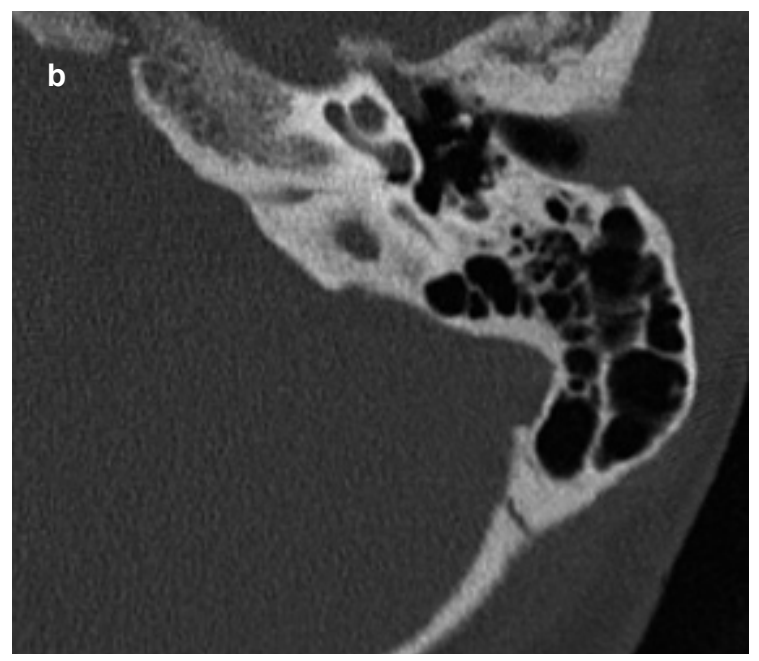

FIG. 1. Axial computed tomography of both cochlea with no sign of labyrinthitis ossificans
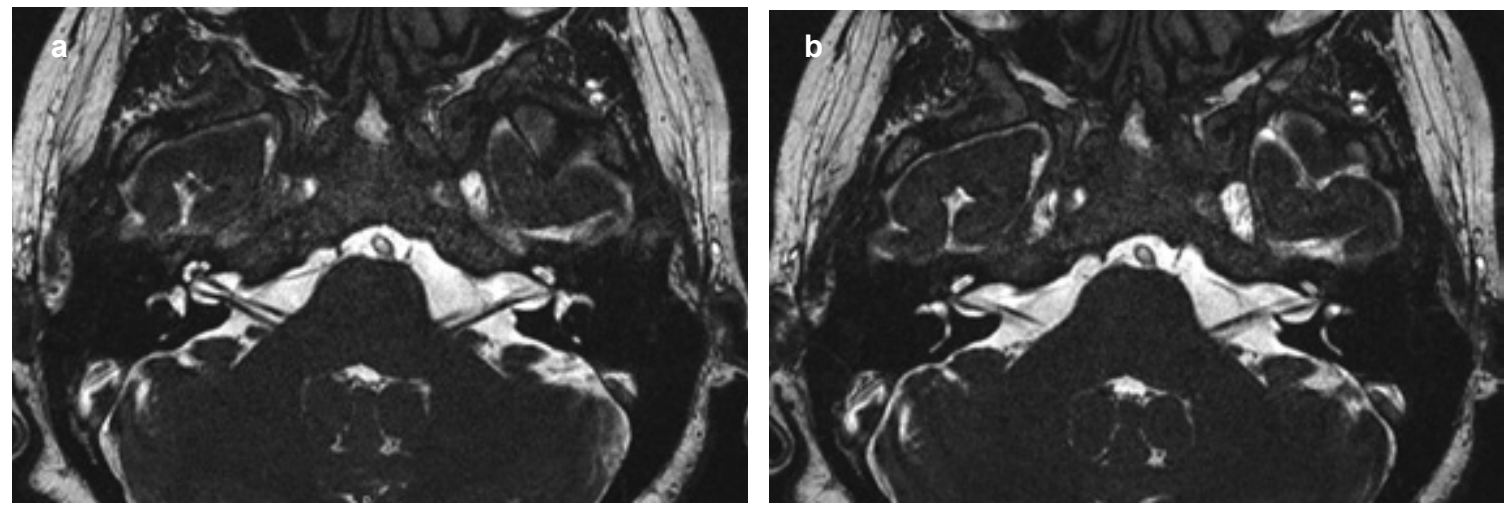

FIG. 2. Axial T2 weighted MR images showing intact scala tympani and vestibule with normal cochlear fluid distribution with no sign of labyrinthitis ossificans 


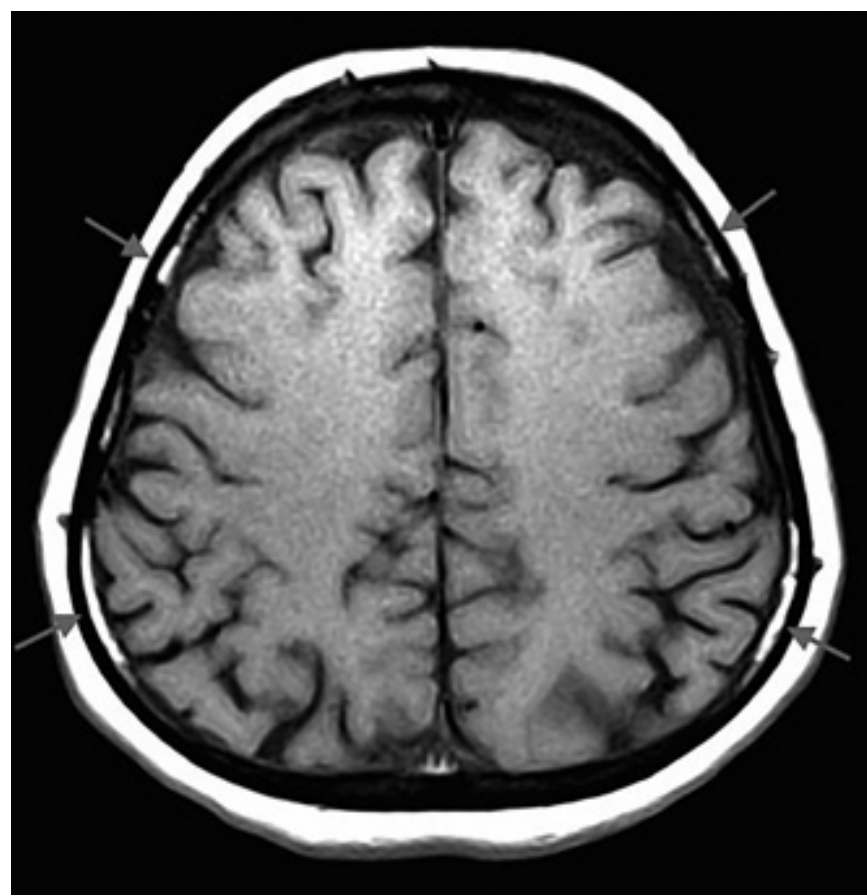

FIG. 3. Cranial MRI showing diffuse dural thickening and minimal diffuse pial contrast enhancement consistent with meningitis (arrows)

On follow-up, her medical status was greatly improved with the obvious exception of hearing loss. Her control cranial MRI in June 2014 showed regression in dural thickening and pial contrast enhancement (Figure 4). With approval of the Department of Infectious Diseases, she was operated upon in June 2014. With short post auricular incision and transmastoid approach, a Nucleus Contour (Cochlear; Sydney, Australia) electrode was inserted on the right side. Intra-operative nerve telemetry tests and postoperative transorbital radiograph confirmed the correct positioning of the electrode. Her mastoid pressure dressing was removed 24 hours later and after the application of the outer part of the cochlear implant, she was discharged from the hospital on the 3rd day. An informed consent was taken from the patient.

\section{Auditory perception skills}

Auditory perception skills were evaluated with categories of auditory perception (CAP) and Sentence Recognition Test in Turkish. She can discriminate environmental sounds and speech sounds. Her CAP score reached 5, which is "Understanding of common phrases without lip reading". The patient started to understand one on one conversations, but was still struggling to participate in conversations with more than one person. Also, as expected, noisy situations were challenging for her to understand spoken words. Although discriminating melodies was not easy, she reported that she listens to music. In the sentence recognition test, she correctly repeated $10 \%$ of

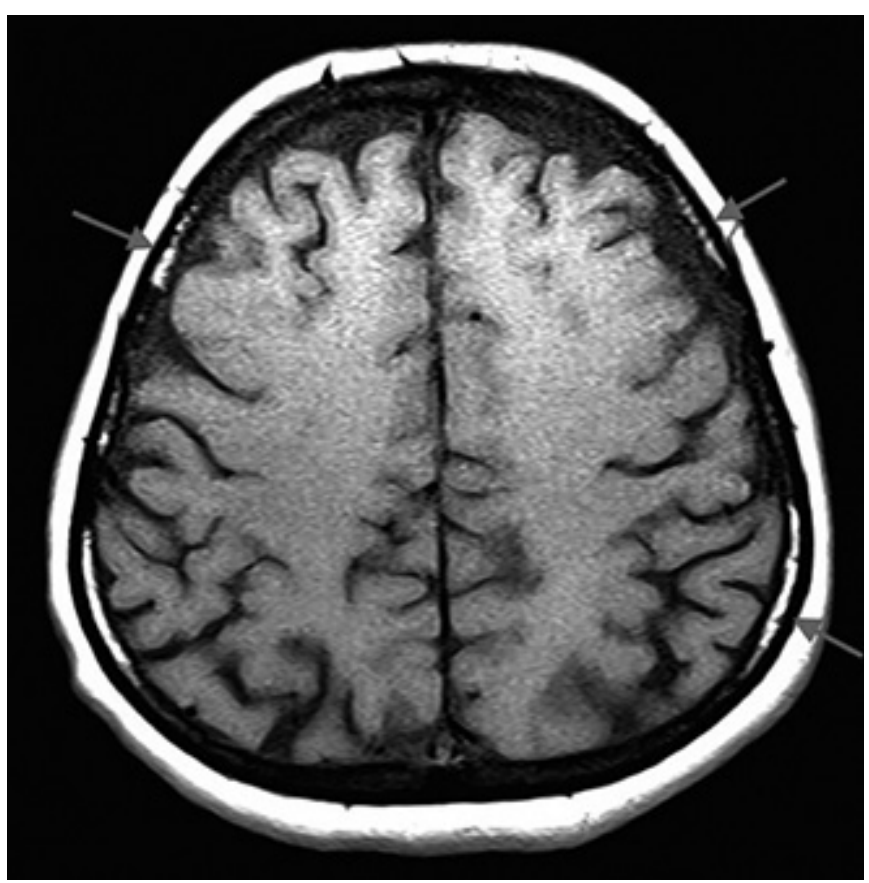

FIG. 4. Cranial MRI showing with regression in dural thickening and pial contrast enhancement (arrows)

sentences in auditory-verbal condition. This result is compatible with her CAP score, because she only understood common phrases in the test.

\section{Speech intelligibility skills}

Her speech intelligibility skills are still improving. Her primary language is Kurdish and her husband reports that her Speech Intelligibility Rating (SIR) score is much better in Kurdish. According to clinical observations and statements from her family members, her SIR score is 3 . This indicates that "in connected speech, her speech is intelligible only if the listener concentrates hard. However, the listener may need to make use of lip-reading/contextual cues." In oneon-one conversations, if the context is familiar to her and the listener, the communication process is much better.

\section{DISCUSSION}

Temporary SNHL in the acute systemic form of brucellosis and permanent hearing loss in neurobrucellosis were previously reported $(2,3)$, while a review of 32 patients with brucellosis showed no abnormalities of hearing status (4). Overall, hearing evaluation is recommended in all forms of the disease (5). Considering deficits like limb weakness, peripheral and central neuropathies, diplopia, ataxia, and paralysis, SNHL 
increases the burden of disease and needs to be addressed thoroughly. In a previous observational study, hearing loss was found to be more prevalent in patients with dementia and cognitive impairment compared to control subjects in an older adult population; however, a causal relationship could not be established (5). Hearing aids are the mainstay of rehabilitation and in select patients with profound hearing loss, cochlear implantation would be an appropriate intervention. A previous study, as well as ours, suggests good outcome with cochlear implantation (6). Patients in both of these studies showed no sign of labyrinthitis ossificans, unlike the more common pneumococcal meningitis. When outcomes of cochlear implantation in adults older than 60 years were analyzed, post-cochlear implantation hearing-in noise test (CI HINT) scores were significantly improved after the operation. Individuals with higher pre-CI HINT scores had significantly greater post-CI HINT score improvement (40-60\%) than those with lower pre-CI HINT scores ( $<40 \%$ improvement) after adjusting for age at hearing loss onset (7). The same study also showed that the gain in speech scores was negatively affected as the time interval between the hearing loss onset and the timing of cochlear implantation. Cost-utility concerns are raising more than ever in the current era of expensive healthcare systems and cochlear implantation is not an exception. Comparing 229 cochlear implant patients with 32 individuals waiting for cochlear implantation in terms of quality-adjusted life-years, the cost-utility benefits of the devices were favorable compared with other medical interventions. Similar studies revealed that there were significant increases in speech perception and quality of life scores after surgery and cochlear implantation is a feasible rehabilitation procedure for patients with profound hearing loss. Gaylor et al. (8) performed a systematic review about cochlear implantation in adults, and showed that most of the unilateral CI users show improved speech recognition skills. In cases such as ours, cochlear implant surgery must be performed immediately since cross-modal plasticity was not observed in post-lingual adults in the study by Buckley and Tobey (9) and auditory deprivation affects speech perception skills.

To the best of our knowledge, our report represents the second case of neurobrucellosis, in whom bilateral profound SNHL had developed, which was successfully treated with cochlear implantation with good functioning hearing results postoperatively.

\section{Ethics Committee Approval: N/A.}

Informed Consent: Written informed consent was obtained from patient who participated in this study.
Peer-review: Externally peer-reviewed.

Author contributions: Concept - Ö.S., M.D.B., F.A.; Design Ö.S., M.D.B., F.A.; Supervision - D.B., L.S.; Resource - D.B., L.S.; Materials - Ö.S., F.A.; Data Collection \&/or Processing - Ö.S., F.A., D.B.; Analysis \&/or Interpretation - Ö.S., F.A., D.B.; Literature Search - Ö.S., M.D.B., F.A.; Writing - Ö.S., F.A., M.D.B.; Critical Reviews - M.D.B., Ö.S., F.A.

Conflict of Interest: No conflict of interest was declared by the authors.

Financial Disclosure: The authors declared that this study has received no financial support.

\section{REFERENCES}

1. Gul H, Erdem H, Bek S. Overview of neurobrucellosis: a pooled analysis of 187 cases. Int J Infect Dis 2009;13:e339-e43. [CrossRef]

2. Kaygusuz TO, Kaygusuz I, Kilic SS, Yalcin S, Felek S. Investigation of hearing loss in patients with acute brucellosis by standard and high-frequency audiometry. Clin Microbiol Infect 2005;11:559-63. [CrossRef]

3. Guven T, Ugurlu K, Ergonul O, Celikbas AK, Gok SE, Comoglu $\mathrm{S}$, et al. Neurobrucellosis: Clinical and Diagnostic Features. Clin Infect Dis 2013;56:1407-2. [CrossRef]

4. Bayazit YA, Namiduru M, Bayazit N, Ozer E, Kanlikama M. Hearing status in brucellosis. Otolaryngol Head Neck Surg 2002;127:97-100. [CrossRef]

5. Uhlmann RF, Larson EB, Rees TS, Koepsell TD, Duckert LG. Relationship of hearing impairment to dementia and cognitive dysfuction in older adults. JAMA 1989;261:1916-9. [CrossRef]

6. Guneri E, Kirkim G, Serbetcioglu B, Erdag T, Guneri A. Cochlear Implantation in Neurobrucellosis. Otol Neurotol 2009;30:747-9. [CrossRef]

7. Lin FR, Chien WW, Lingsheng Li, Niparko JK, Francis HW. Cochlear implantation in older adults. Medicine (Baltimore) 2012;91:229-41. [CrossRef]

8. Gaylor JM, Raman G, Chung M, Lee J, Rao M, Lau J, et al. Cochlear Implantation in Adults: A Systematic Review and Metaanalysis. JAMA Otolaryngol Head Neck Surg 2013;139:265-72. [CrossRef]

9. Buckley K, Tobey E. Cross-Modal Plasticity and Speech Perception in Pre- and Postlingually Deaf Cochlear Implant Users. Ear Hear 2010:1. [CrossRef] 